\title{
Implantable Distributed Biomedical Photonic Devices
}

\author{
Jun Ohta*, Ayato Tagawa, Takuma Kobayashi, Toshihiko Noda, \\ Kiyotaka Sasagawa and Takashi Tokuda \\ Graduate School of Materials Science, Nara Institute of Science and Technology, \\ 8916-5 Takayama, Ikoma, Nara 630-0101, Japan
}

(Received January 13, 2011; accepted February 17, 2011)

Key words: biomedical photonic devices, complementary metal-oxide-semiconductor (CMOS) image sensor, implantable device, distributed implantation, brain imaging

In this paper, we demonstrate an implantable distributed biomedical photonic device that is based on a dedicated complementary metal-oxide-semiconductor (CMOS) image sensor architecture to measure fluorescence by implantation into the deep brain of an untethered mouse. To reduce tissue damage during implantation, an ultrasmall chip is fabricated using a standard $0.35 \mu \mathrm{m}$ CMOS technology. The chip has an area of 547 $\times 700 \mu \mathrm{m}^{2}$ and $60 \times 60$ pixels and is demonstrated to capture images. An implantable device is fabricated using three microchips, and the fundamental operation of the device is validated. The device successfully detected fluorescence from fluorescent beads excited externally. In addition, the device achieved simultaneous operation of the three microchips with a delay of less than $15 \mu \mathrm{s}$.

\section{Introduction}

The rapid development of optical and electrical imaging methods to measure brain activities, such as fluorescence microscopy including two-photon microscopy, electrocorticogram (ECoG), positron emission tomography (PET), functional magnetic resonance imaging (fMRI), functional near-infrared spectroscopy (fNIRS) or optical tomography, has brought about significant advancements in brain science and technology. ${ }^{(1)}$ Recently, attempts have been reported to apply some of these measurement methods to small experimental animals, such as the mouse and the rat, in an untethered environment. ${ }^{(2-5)}$ However, these methods have yielded inadequate results owing to, for example, insufficient spatial resolution for measuring small brains, such as the mouse brain, ${ }^{(2)}$ or the weight and/or bulk of the devices being too great, making it difficult for a mouse implanted with a device to move freely. ${ }^{(4,5)}$

*Corresponding author: e-mail: ohta@ms.naist.jp 
By solving these problems, we have developed a biomedical photonic device that can be implanted in the mouse deep brain and can measure brain activities through the fluorescence of specific neural activities at a spatiotemporal resolution of less than 100 $\mathrm{ms}$ and less than $100 \mu \mathrm{m} .{ }^{(6-10)}$ The basic configuration of the device is shown in Fig. 1 , in which the device is implanted into the deep brain of a mouse. Table 1 compares

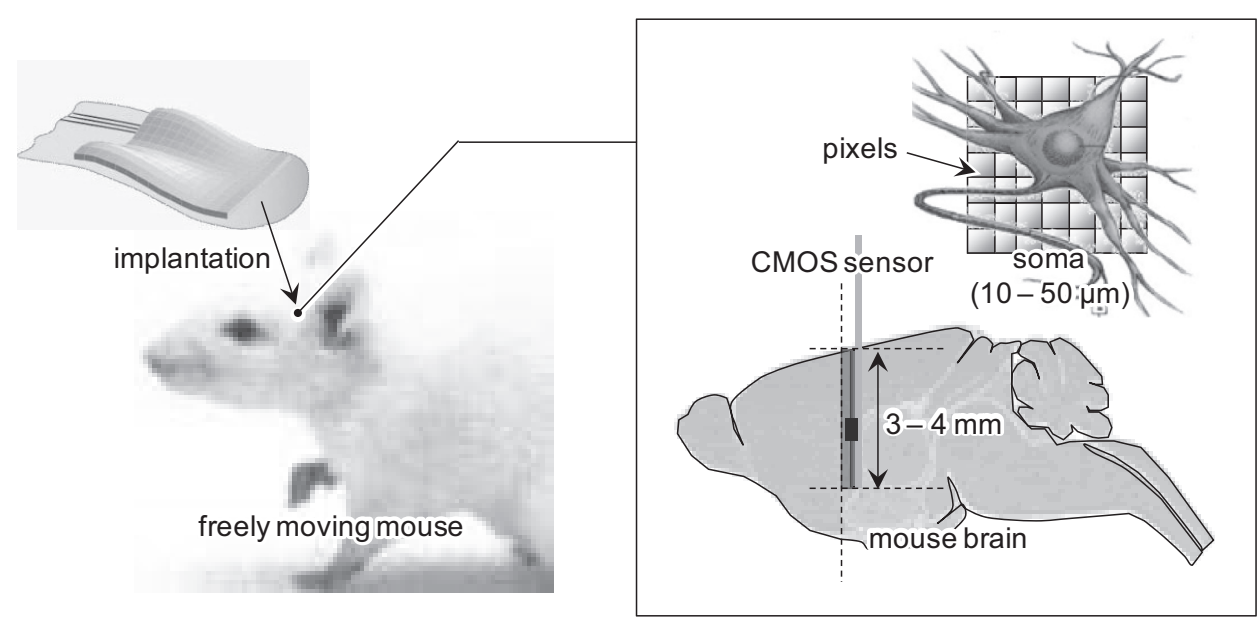

Fig. 1. Conceptual illustration of the biomedical photonic LSI.

Table 1

Brain imaging methods for a freely moving mouse.

\begin{tabular}{|c|c|c|c|c|c|}
\hline Method & Invasiveness & Observation subject & $\begin{array}{l}\text { Measurement depth } \\
\text { in brain }\end{array}$ & $\begin{array}{l}\text { Time response } \\
\text { (s) }\end{array}$ & $\begin{array}{l}\text { Spatial resolution } \\
(\mathrm{mm})\end{array}$ \\
\hline fMRI & Yes & Blood flow rate & Arbitrary depth & $\sim 1$ & $\sim 1$ \\
\hline PET & Yes & $\begin{array}{c}\text { Consumption of glucose/ } \\
\text { oxygen }\end{array}$ & Arbitrary depth & $\sim 10$ & $\sim 10$ \\
\hline fNIRS/optical topography & Yes & $\begin{array}{l}\text { Oxidation/reduction of } \\
\text { hemoglobin }\end{array}$ & Brain surface & $\sim 0.1$ & $\sim 1$ \\
\hline ECoG & No & Electrical potential & Brain surface & $\sim 0.001$ & $\sim 1$ \\
\hline Fluorescence microscopy & No & $\begin{array}{c}\text { Fluorescence } \\
\text { (molecular imaging) }\end{array}$ & Brain surface & $\sim 0.001$ & 0.001 \\
\hline $\begin{array}{l}\text { Two-photon excited } \\
\text { fluorescence microscopy }\end{array}$ & No & $\begin{array}{c}\text { Fluorescence } \\
\text { (molecular imaging) }\end{array}$ & $\begin{array}{l}\text { Brain surface } \\
\quad(\sim 1 \mathrm{~mm})\end{array}$ & $\sim 0.001$ & 0.001 \\
\hline This work & No & $\begin{array}{c}\text { Fluorescence } \\
\text { (molecular imaging) }\end{array}$ & Arbitrary depth & $\sim 0.01$ & 0.01 \\
\hline
\end{tabular}


the proposed device and previously reported devices from the viewpoints of spatial and temporal resolutions, as well as other criteria. The proposed device is based on a complementary metal-oxide-semiconductor (CMOS) image sensor with a multimodal sensing function, which includes the measurement of neural potentials and the simulation of neuron cells..$^{(9,10)}$ Since CMOS image sensors can be integrated into functional circuits on the chip, such multimodal sensing can be realized. We have successfully demonstrated LTP activities in the mouse hippocampus using a biomedical photonic device. ${ }^{(7)}$ In addition, we have demonstrated that a mouse with the proposed device implanted in the deep brain can move freely. ${ }^{(8)}$ Although the proposed device is invasive, damage to brain tissues is relatively minimal. However, it is preferable to reduce such damage.

As such, we propose the use of multiple small CMOS sensors in order to markedly reduce tissue damage. Several small sensor chips are implanted in the mouse brain in a distributed manner. The captured fluorescence image is synthesized in order to obtain a complete image.

In the next section, we describe the materials and methods used to implement the ultrasmall CMOS sensors. We then present experimental results and discuss future concerns. Conclusions are presented in the final section.

\section{Materials and Methods}

\subsection{Ultrasmall CMOS sensor}

We have fabricated an ultrasmall CMOS sensor using a standard $0.35 \mu \mathrm{m}$ twopoly four-metal CMOS technology designed by Austria Micro Systems (AMS). The specifications and a block diagram of the chip are shown in Table 2 and Fig. 2, respectively. The sensor architecture is based on a standard CMOS image sensor with a

Table 2

Specifications of the chip.

\begin{tabular}{|c|c|c|}
\hline \multicolumn{2}{|c|}{ Technology } & $0.35 \mu \mathrm{m}$ 2-poly 4-metal CMOS process \\
\hline \multicolumn{2}{|c|}{ Operating voltage } & $3.3 \mathrm{~V}$ \\
\hline \multicolumn{2}{|c|}{ Circuit size } & $547 \times 700 \mu \mathrm{m}^{2}$ \\
\hline \multirow{6}{*}{ Pixel } & Number & $60 \times 60$ \\
\hline & Pixel size & $7.5 \times 7.5 \mu \mathrm{m}^{2}$ \\
\hline & Array size & $450 \times 450 \mu \mathrm{m}^{2}$ \\
\hline & Type & 3-Transistor APS \\
\hline & Photodiode & n-well/p-sub \\
\hline & Fill factor & $35 \%$ \\
\hline
\end{tabular}




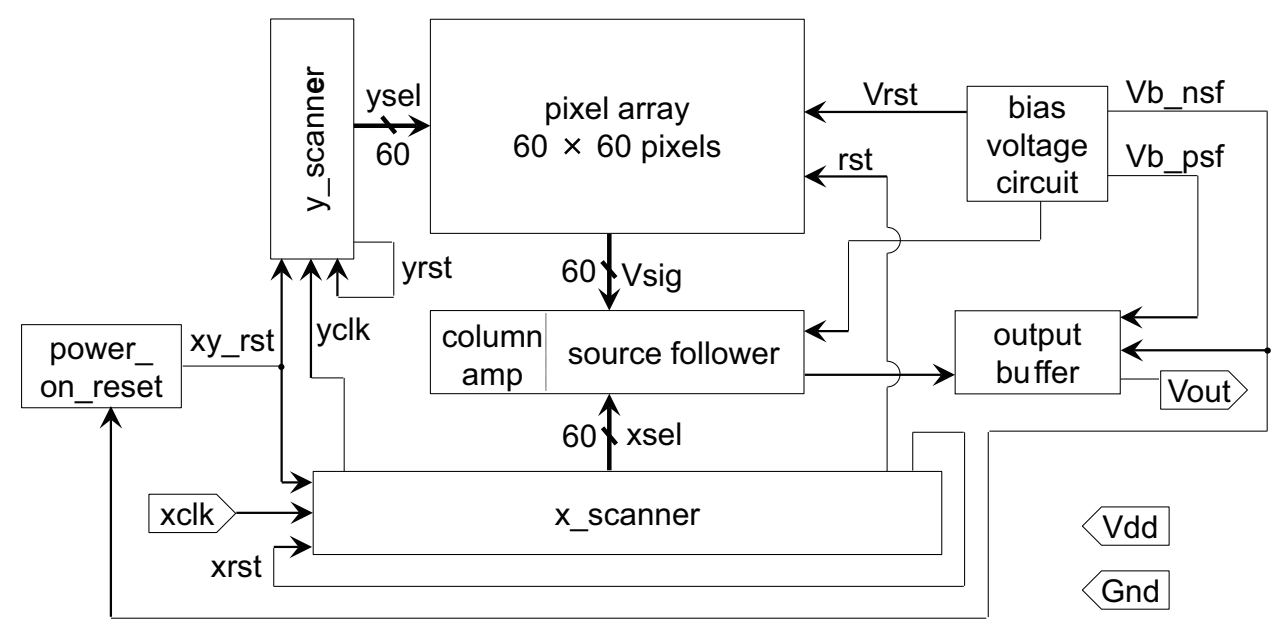

Fig. 2. Block diagram of the ultrasmall CMOS sensor.

three-transistor active pixel sensor (3T-APS) ${ }^{(11)}$ that has been modified for implantation into the deep brain of a mouse, which can move freely. For this purpose, input/output (IO) pads are placed on one end of each chip for easy insertion of the device deep into the mouse brain, and the number of IOs is decreased to four in order to facilitate free movement of the mouse after implantation. ${ }^{(8)}$

To minimize tissue damage during implantation, we further modified the chip design as follows:

(i) The number of pixels is decreased from $120 \times 268$ to $60 \times 60$ pixels, for an imaging area of $450 \times 450 \mu \mathrm{m}^{2}$, which can cover a number of neuron cells.

(ii) Sample and hold circuits integrated in column amplifiers are omitted.

(iii) A single small bias circuit is designed to produce the required bias voltage, whereas the former chip has four bias circuits.

Figures 3(a) and 3(b) show the bias circuits and their layout. A high-resistive polysilicon layer is used as a resistor in order to reduce the resistor area. The resistor is composed of an array of $2 \times 9$ resistor units, each of which has the same shape and resistance value. Three bias voltage nodes of Vrs, Vbp, and Vbn are tapped from the resistor. Dummy resistors having the same shape are placed at the bottom and top of the resistor array. Although the poly-silicon resistor layer has poor precision, the bias voltage is determined only by the resistance ratio and so is less affected by resistance variation.

As shown in Fig. 3(c), the bias circuit was placed under the bonding pads, Vdd and GND, where the voltage is constant, which reduces the effect on the bias circuit. To avoid incorrect operation induced by an unintentionally large input current, small electrostatic discharge circuits are placed under the bonding pads for Vout and xclk, as shown in Fig. 3(c). 


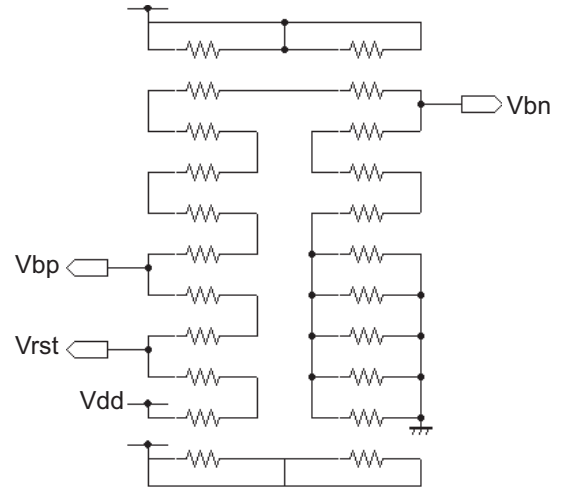

(a)

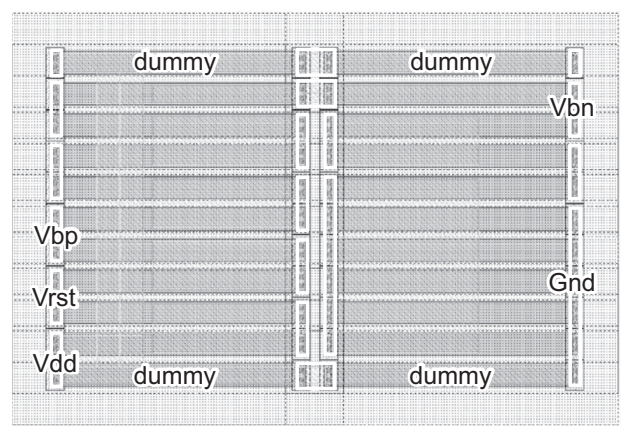

(b)

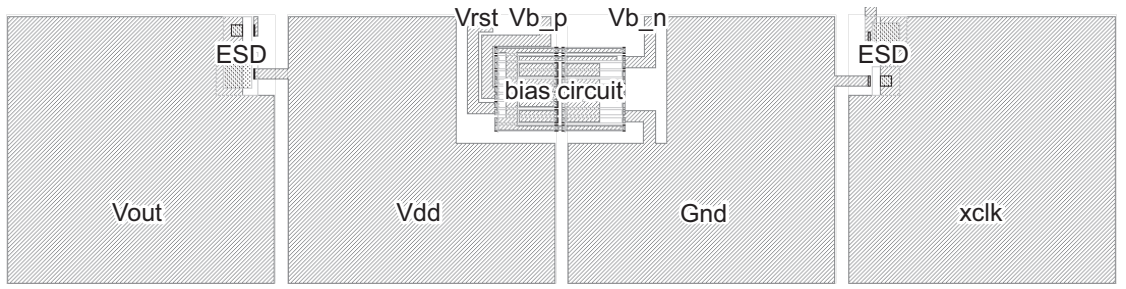

(c)

Fig. 3. Bias circuits. (a) Circuits, (b) layout, and (c) placement of circuits under the pad layer.

A photomicrograph of the fabricated chip is shown in Fig. 4. The total chip area is reduced to $547 \times 700 \mu \mathrm{m}^{2}$, which is $10 \%$ smaller than the previously developed CMOS sensor chip. ${ }^{(8)}$ The chip is divided into microchips using a deep reactive etching machine by the Bosch process. This process enables a chip to be divided with little damage and high precision. A camera system is constructed to test the operation of the chip, as shown in Fig. 5. A lens (Fujinon, YV2.7×2.9LR4D-2) is placed in front of the chip mounted on a pin grid array (PGA) package. The chip is connected through four wires, Vdd, xclk, Vout, and GND, to a control board.

\subsection{Assembly and control system for multiple ultrasmall CMOS sensors}

Three fabricated microchips are assembled on a flexible polyimide substrate, as shown in Fig. 6. The substrate is divided into three parts to accommodate three chips. Each chip is connected to the substrate through four wires of $60 \mu \mathrm{m}$ width and $100 \mu \mathrm{m}$ pitch. The surface of the assembled chip is covered with a green filter of $2 \mu \mathrm{m}$ thickness. The entire device is coated with a parylene coating of $3 \mu \mathrm{m}$ thickness to protect against water as well as to ensure biocompatibility. The assembled device is small and flexible and can be implanted into the mouse deep brain. 


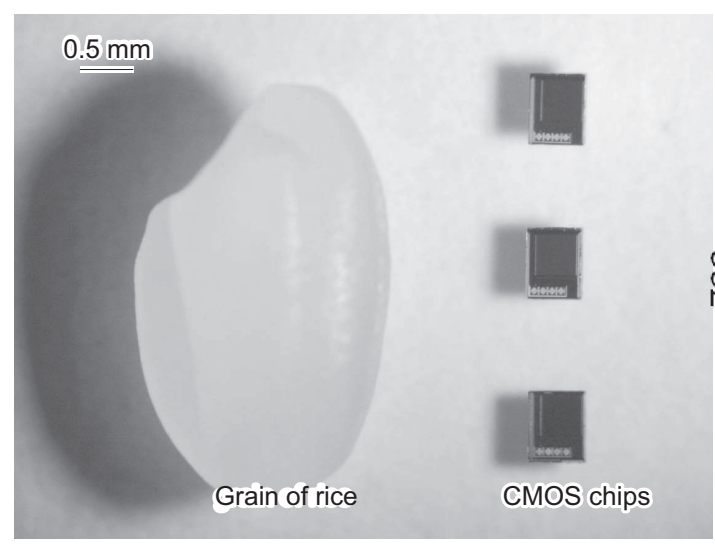

(a)

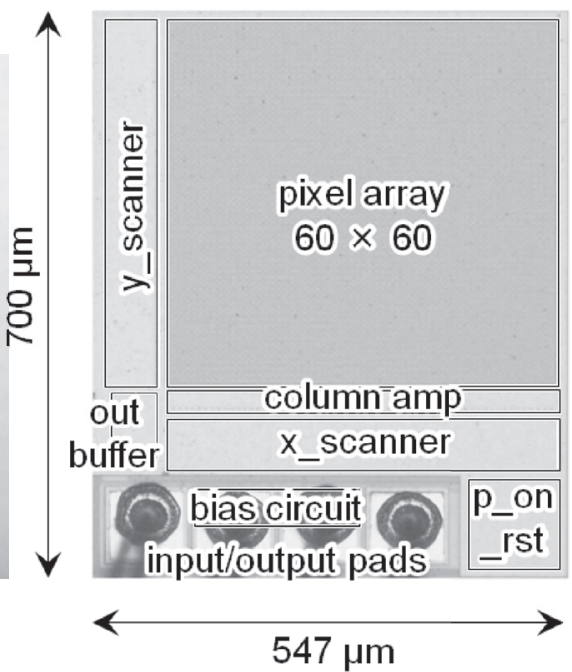

(b)

Fig. 4. Images of the microchips. (a) Three microchips placed next to a grain of rice for size comparison. (b) Close-up view of the microchip.

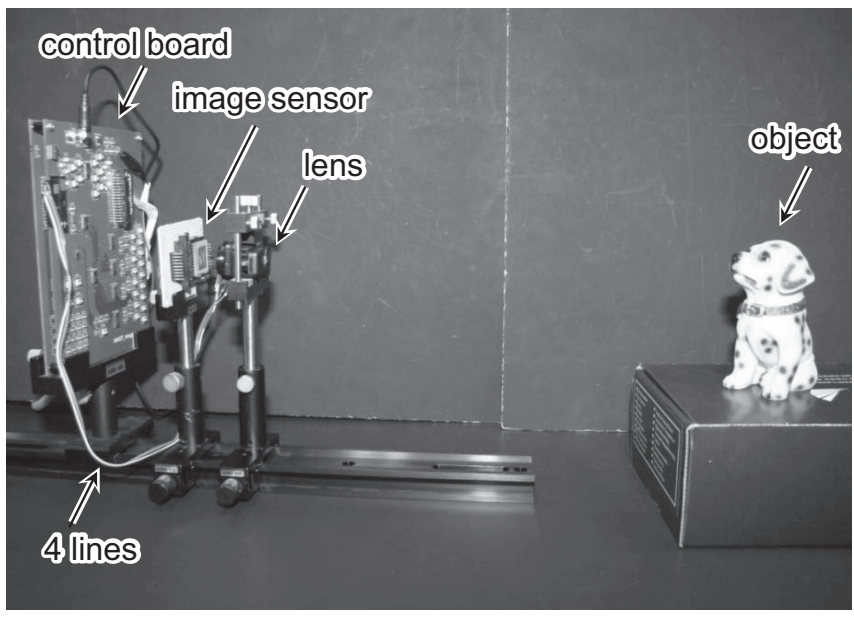

(a)

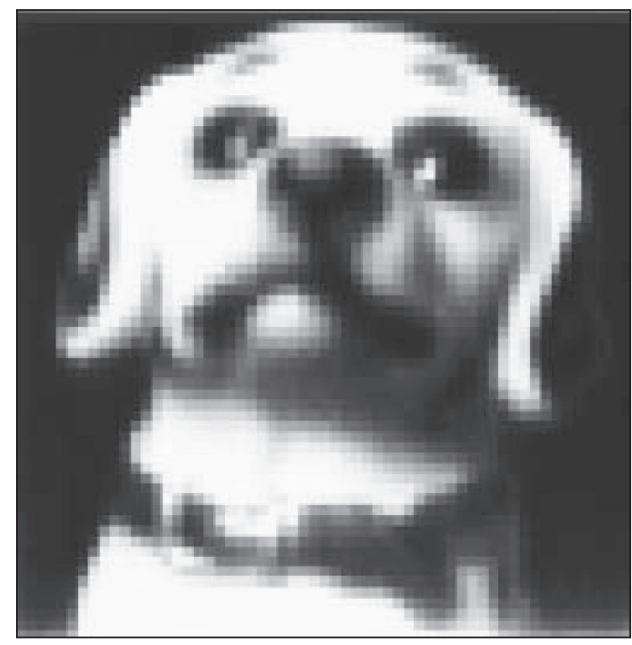

(b)

Fig. 5. Camera system installed with the microchip. (a) Setup of the camera system. (b) Image captured by the camera system. 


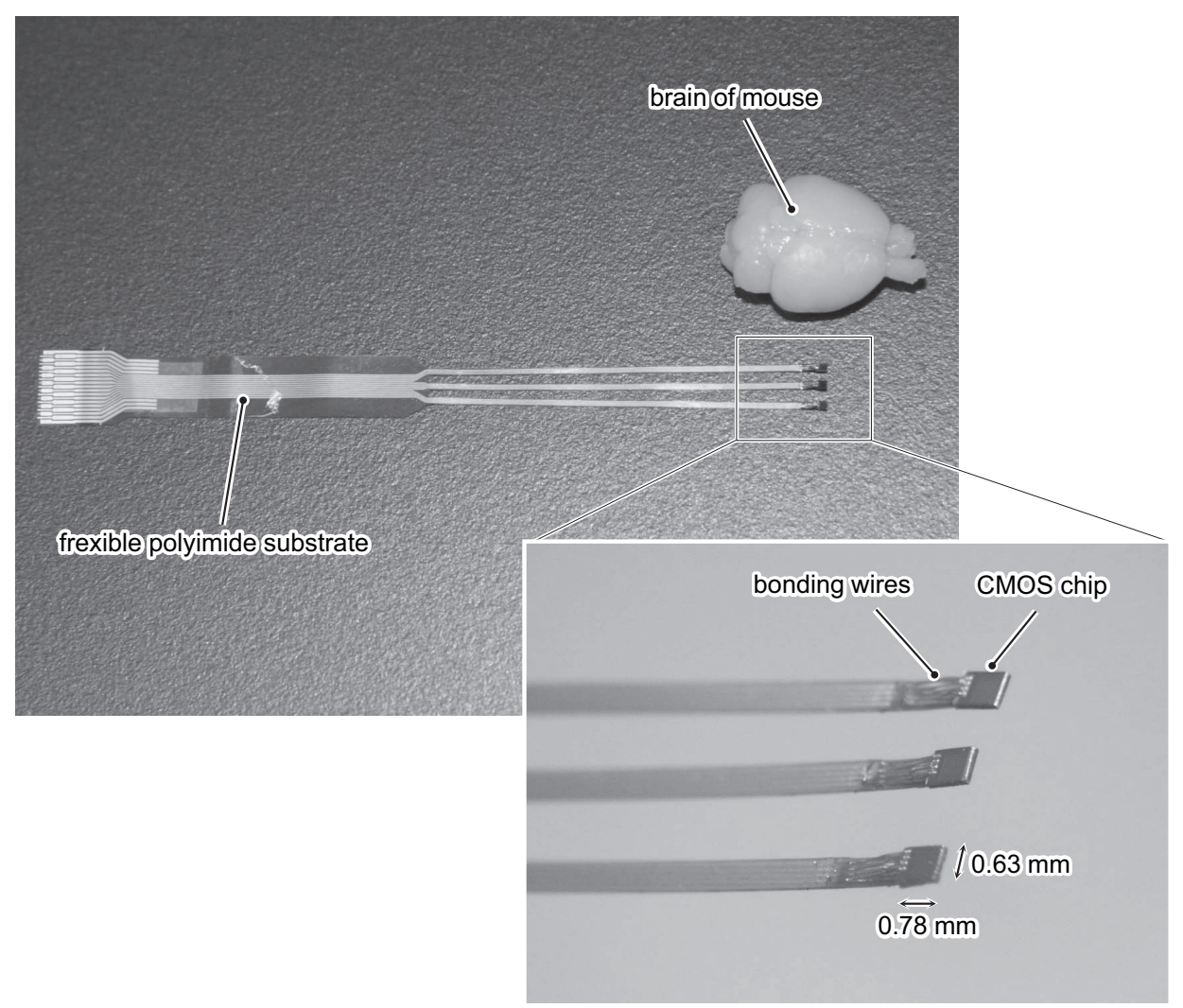

Fig. 6. Photograph of the assembled device.

We have designed and fabricated a control system for three ultrasmall CMOS sensors. The system enables three chips to capture images almost simultaneously in less than $1 \mathrm{~s}$. The block diagram of the system is shown in Fig. 7, where three PCI control boards are installed in one computer. A photograph of the system is shown in Fig. 8.

\section{Results and Discussion}

First, the chip was tested using the camera system described in $\S 2.1$. A captured image is shown in Fig. 5(b). One of the main concerns in the chip fabrication process is the possibility of damaging the circuits placed under the pads when the wire bonding process is performed. Thermal and/or ultrasonic shocks may be introduced during the process. Ten chips were tested and were found to have almost the same characteristics. Thus, the wire bonding process does not affect the characteristics of the circuits.

Next, the assembled device, as described in $\S 2.2$, was tested for simultaneous 


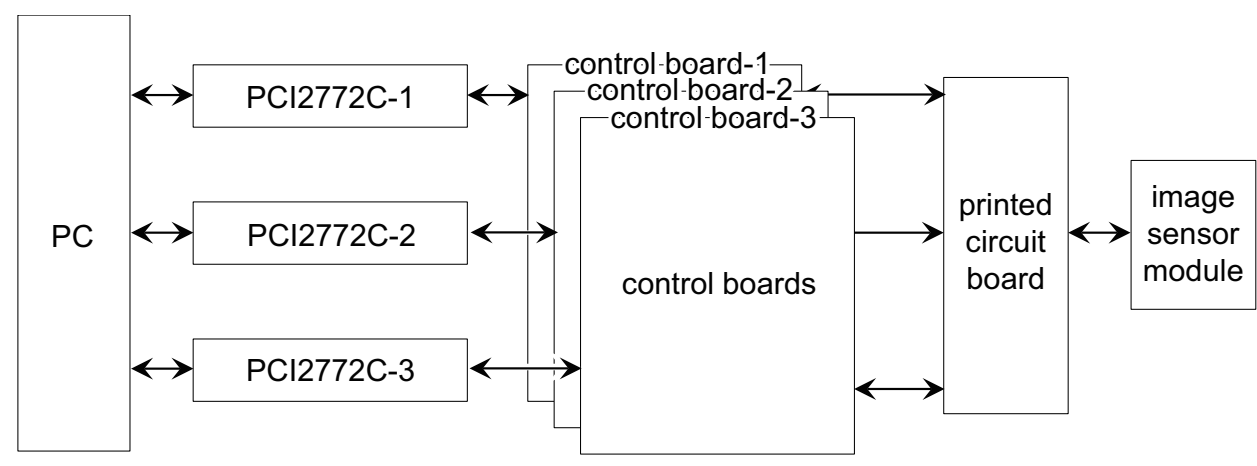

Fig. 7. Block diagram of the control system.

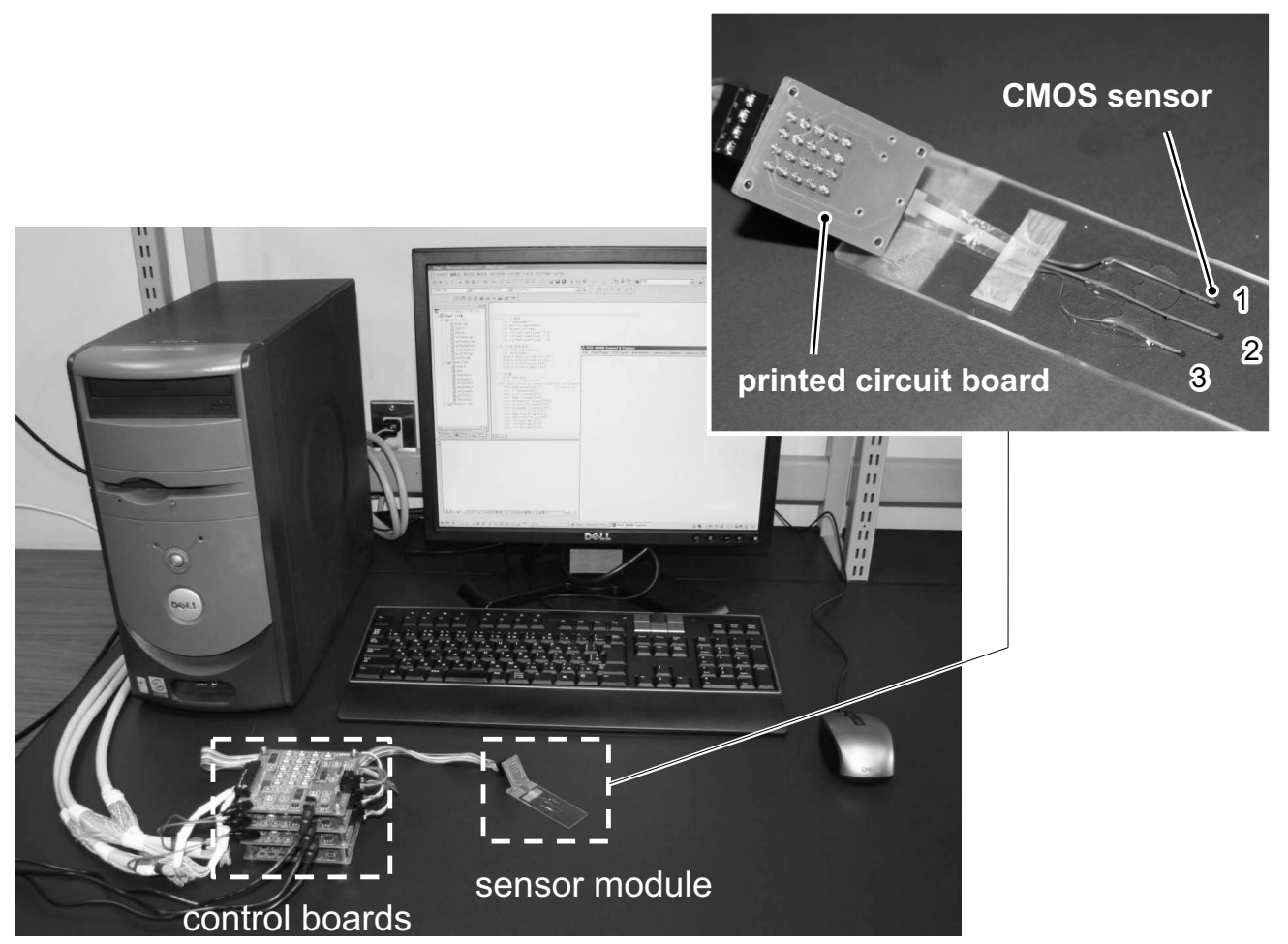

Fig. 8. Photograph of the experimental system. 
imaging using fluorescent beads. Beads (Fluoresbrite ${ }^{\circledR}$ YG Microspheres, Polysciences) having diameters of 45,20 , and $10 \mu \mathrm{m}$ were distributed on the surface of the imaging area and were captured by the sensor chips under the illumination of a fluorescence excitation light source. We successfully captured images of the beads during the simultaneous operation of the three chips, as shown in Fig. 9.

Figure 10 shows the input and output signals to and from the device. The output signals from three microchips for 60 pulses input to scan one column are shown in the figure. The output signals from all three microchips are saturated in this case. Each sensor can be operated in 60 frames per second. The time lag between the two sensor output signals was only $15 \mu$ s. This time lag is usually neglected in brain neural imaging.

Wireless operation is preferable for the measurement of freely moving mice. As such, several candidates for wireless operation, including RF and infrared light techniques, are expected. The requirements for an implantable device are small chip area, which
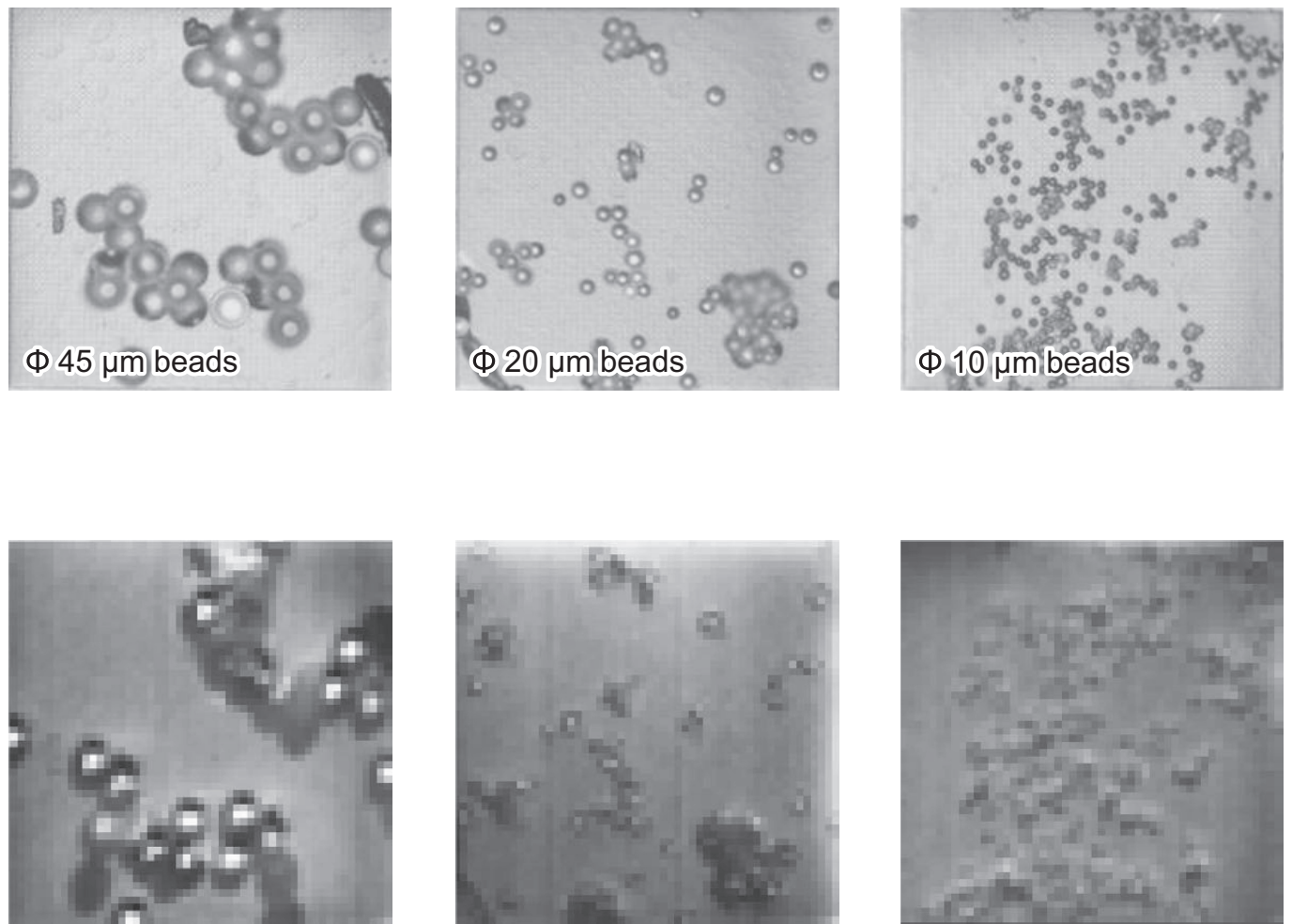

Fig. 9. Captured images of fluorescent beads distributed on the chip. The upper three photographs are bright images captured by optical microscopy, and the lower three photos are fluorescent images captured by the sensor. 


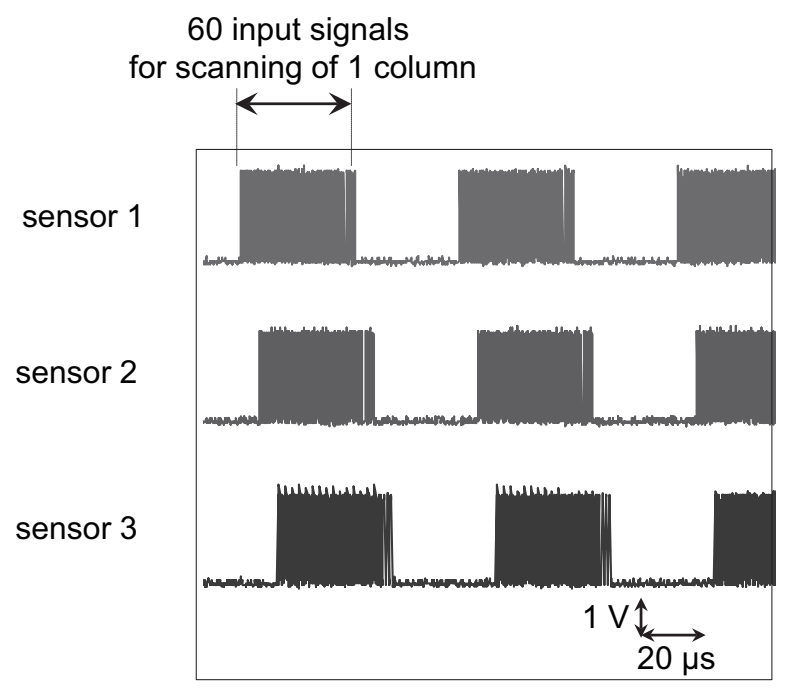

(a)

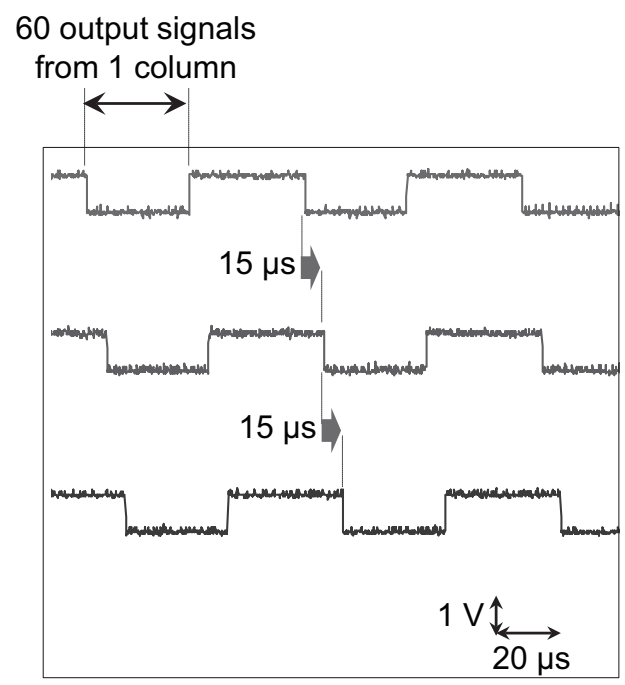

(b)

Fig. 10. Input (a) and output (b) signal waveforms of the three microchips.

would include the RF coil and the infrared light detector, and small power consumption. Note that wireless operation includes power transmission as well as data transmission. In some cases, wireless power transmission and data transmission should be designed separately. For example, large-format image data, such as VGA signals, require highspeed transmission, whereas the power supply line does not require such high-speed transmission. In such cases, power is transmitted optically, while data is transmitted electrically.

If the distributed chips are placed on the surface of the brain, other communication methods, such as capacitive coupling, are expected because the implanted device can be placed close to the detection or transmission system. Further study is required to develop effective implantable systems.

\section{Conclusions}

We have successfully demonstrated a distributed implantable device for measuring mouse brain activities in order to reduce tissue damage during implantation. The device is based on CMOS image sensor architecture and is fabricated using a $0.35 \mu \mathrm{m}$ standard CMOS technology. Three microchips are assembled in one polyimide substrate and are operated simultaneously. The time lag between chips is only $15 \mu \mathrm{s}$. In the future, the proposed device will be validated in vivo and will be equipped with wireless communications and a power supply. 


\section{Acknowledgments}

The present study was supported in part by the Japan Agency of Science and Technology (JST), Core Research Evolutional Science and Technology (CREST), and the Research Seed Quest Program. The authors would like to thank Prof. Sadao Shiosaka of the Graduate School of Biological Sciences, Nara Institute of Science and Technology, Japan, for the discussion from the viewpoint of bioscience.

\section{References}

1 J. C. Mazziota: Time and Space in Brain Mapping Methods, eds. A. W. Toga and J. C. Mazziota (Academic Press, New York, 2002) p. 33.

2 C. Woody, A. Kriplani, P. O’Connor, J. F. Pratte, V. Radeka, S. Rescia, D. Schlyer, S. Shokouhi, S. Stoll, P. Vaska, A. Villaneuva, N. Volkowa and B. Yu: Nucl. Instrum. Methods Phys. Res. A, $\mathbf{5 2 7}(2004) 166$.

3 M. Lee, H. S. Shin and J. H. Choi: Proc. Annual International Conference of the IEEE Engineering in Medicine and Biology Society (IEEE, New York, 2009) p. 2934.

4 F. Helmchen, M. S. Fee, D. W. Tank and W. Denk: Neuron 31 (2001) 903.

5 J. H. Park, V. Pieribone, D. Kim, J. V. Verhagen, C. V. Hehn and E. Culurciello: Proc. IEEE International Symposium on Circuits and Systems (IEEE, New York, 2009) p. 2429.

6 D. C. Ng, H. Tamura, T. Tokuda, A. Yamamoto, M. Matsuo, M. Nunoshita, Y. Ishikawa, S. Shiosaka and J. Ohta: J. Neurosci. Methods 156 (2006) 23.

7 H. Tamura, D. C. Ng, T. Tokuda, H. Naoki, T. Nakagawa, T. Mizuno, Y. Hatanaka, Y. Ishikawa, J. Ohta and S. Shiosaka: J. Neurosci. Methods 173 (2008) 114.

8 A. Tagawa, A. Higuchi, T. Sugiyama, K. Sasagawa, T. Tokuda, H. Tamura, Y. Hatanaka, S. Shiosaka and J. Ohta: Jpn. J. Appl. Phys. 48 (2009) 04C195-1.

9 A. Tagawa, H. Minami, M. Mitani, T. Noda, K. Sasagawa, T. Tokuda, H. Tamura, Y. Hatanaka, Y. Ishikawa, S. Shiosaka and J. Ohta: Jpn. J. Appl. Phys. 49 (2010) 01 AG02.

10 A. Tagawa, M. Mitani, H. Minami, T. Noda, K. Sasagawa, T. Tokuda and J. Ohta: Jpn. J. Appl. Phys. 49 (2010) 04DL02.

11 J. Ohta: Smart CMOS Image Sensors and Applications (CRC Press, Boca Raton, FL, 2007) Chap. 2. 